\title{
Anisotropic Jüttner (relativistic Boltzmann) distribution
}

\author{
Rudolf A. Treumann ${ }^{1, a}$ and Wolfgang Baumjohann ${ }^{2}$ \\ ${ }^{1}$ Department of Geophysics and Environmental Sciences, Munich University, Munich, Germany \\ ${ }^{2}$ Space Research Institute, Austrian Academy of Sciences, Graz, Austria \\ ${ }^{\mathrm{a}}$ currently at: International Space Science Institute, Bern, Switzerland
}

Correspondence to: Rudolf A. Treumann (art@geophysik.uni-muenchen.de)

Received: 15 July 2016 - Revised: 26 August 2016 - Accepted: 31 August 2016 - Published: 7 September 2016

\begin{abstract}
A rigorous derivation of the Jütner (covariant Boltzmann) distribution is provided for anisotropic pressure (or temperature) tensors. It was in similar form anticipated first by Gladd (1983). Its manifestly covariant version follows straightforwardly from its scalar property.
\end{abstract}

Keywords. Space plasma physics (kinetic and MHD theory)

The Jüttner distribution (Jüttner, 1911) is the relativistically generalized classical isotropic Maxwell-Boltzmann distribution, whether written in its dependence on relativistic particle energy $\epsilon_{\boldsymbol{p}}=m \gamma(\boldsymbol{p}) c^{2}$ or momentum $\boldsymbol{p}$, with $\gamma=$ $\sqrt{1+p^{2} / m^{2} c^{2}}$. The phase-volume element $\mathrm{d} \boldsymbol{x} \mathrm{d} \boldsymbol{p}$ is covariant (a consequence of its scalar nature). Hence the Jüttner distribution is as well covariant but not manifestly covariant. In anisotropic relativistic gases or plasmas (for application to anisotropic plasmas cf. Yoon, 1989, 2007; Gladd, 1983) and in drifting plasmas (for recent examples cf. e.g. Swisdak, 2013; Lopez et al., 2014; Alves et al., 2015; Zenitani, 2015; DeVore et al., 2015) the form of the Jütner distribution is usually assumed. Below we provide its simple analytical derivation and manifestly covariant version.

Thermally relativistic implies thermal speeds $v_{e} / c \gtrsim 10^{-2}$. Avoiding creation, annihilation, and Compton interactions requires $T<m c^{2}$, with $T$ temperature in energy units, hence weakly relativistic thermal electrons of some $10 \mathrm{eV}<T<$ $0.5 \mathrm{MeV}$, covering most hot classical plasmas.

Maxwell-Boltzmann distributions are solutions of the stationary one-particle Boltzmann equation with the argument of the ratio of the single particle to average thermal energies, viz. $\epsilon_{p} / T$. Properly normalized they give the probability at temperature $T$ for finding all particles of given momentum $\boldsymbol{p}$ (or energy $\epsilon_{\boldsymbol{p}}$ ) in the interval $\mathrm{d} \boldsymbol{p}$ (or $\mathrm{d} \epsilon_{\boldsymbol{p}}$ ) in momentum-space volume $\mathrm{d} \boldsymbol{p}$. With three-momentum vector $\boldsymbol{p}=\left(p_{\perp} \cos \phi, p_{\perp} \sin \phi, p_{\|}\right)$in index notation

$\epsilon_{\boldsymbol{p}}^{2}=c^{2} p_{i} \delta_{j}^{i} p^{j}+m^{2} c^{4}$

suggests introduction of temperature anisotropy guided by the diagonal anisotropy of pressure $\mathbf{P}=N\left[T_{\perp} \delta_{i}^{j}+\right.$ $\left.\left(T_{\|}-T_{\perp}\right) \delta_{3}^{3}\right]$ (as for instance in magnetized plasma), with anisotropy in direction 3 (in plasma the direction of the magnetic field $\boldsymbol{b}=\boldsymbol{B} / \boldsymbol{B}$, for instance). The inverse pressure / temperature tensor is $\mathbf{P}^{-1}=\left(T_{\perp} N\right)^{-1} \boldsymbol{\Theta}$,

$\boldsymbol{\Theta}=\Theta_{j}^{i}=\delta_{i}^{j}+(A-1) \delta_{3}^{3}$, with $A=T_{\perp} / T_{\|}$.

Replacing $\delta_{j}^{i}$ in Eq. (1) with $\Theta_{j}^{i}$, valid in the four-velocity frame $U_{j}=\left(\epsilon_{p} / m c, 0\right)$, defining $\beta_{\perp}=m c^{2} / T_{\perp}, \quad \beta_{\|}=$ $m c^{2} / T_{\|}$, putting $p_{\perp}=p \sin \theta, p_{\|}=p \cos \theta$, and defining $p / m c \longrightarrow p$ yields

$\frac{\epsilon_{\boldsymbol{p}}^{2}}{T_{\perp}^{2}}=\beta_{\perp}^{2}\left[1+p^{2}\left(\sin ^{2} \theta+A \cos ^{2} \theta\right)\right]$.

The square root of Eq. (3) enters the Boltzmann factor. Up to normalization $C$, the anisotropic Jütner distribution function of the ideal gas becomes

$F_{J}(\boldsymbol{p}, A)=C \exp \left\{-\beta_{\perp} \sqrt{1+p_{\perp}^{2}+A p_{\|}^{2}}\right\}$.

With $A=1, \beta_{\perp}=\beta_{\|}=\beta$ this is the ordinary Jüttner function. Expanding the root in the limit $c \rightarrow \infty$ reproduces the ordinary nonrelativistic anisotropic Maxwell-Boltzmann distribution. Extensions to drifting or non-ideal gases are straightforward.

This in principle trivial result was anticipated first without proof by Gladd (1983) in application to the whistler instabil- 
ity in weakly relativistic anisotropic plasmas ${ }^{1}$. Normalization, the purpose of Jüttner's effort, yields

$C=N \sqrt{A} \beta_{\perp} / 4 \pi(m c)^{3} K_{2}\left(\beta_{\perp}\right)$

with $K_{2}\left(\beta_{\perp}\right)$ the Bessel function, trivially containing the anisotropy factor $A$.

The distribution Eq. (4) is covariant, valid in time-like slices of Minkowski space. Explicit manifestly covariant isotropic versions have been provided numerically as well (cf. Chacón-Acosta et al., 2010; Curado et al., 2016). Since $F(\boldsymbol{p})$ is a scalar phase space density, its manifestly covariant version is $F\left(x^{v}, p^{v}\right) \sqrt{-g}$ for both isotropic and anisotropic cases. $g<0$ is the determinant of the metric tensor $g_{\mu v}$ in (+ - - ) metric, a version to be applied in curvilinear coordinates. In general relativistic four-space, $\mu, v=0,1,2,3$, and $\boldsymbol{p} \rightarrow p^{v}$ is the four-momentum. Operator interpretation of the three-momentum $\boldsymbol{p}=\hbar \boldsymbol{k}$ relates any of these versions to quantum field theory.

Jüttner's anisotropic distribution is useful for analytical or numerical (as in Gladd, 1983) calculations. In particle-in-cell simulations the initial distribution is prescribed. In practice there is little need to choose it in the simulations to satisfy the Jüttner equilibrium requirement. Solving for all relativistic particle orbits in their self-consistent fields, the initial distribution readily adjusts itself to the physical distribution that evolves under the mutual interactions.

As a side product, this straightforward rigorous derivation indicates that in relativistic media the isotropic temperature $T$ and its inverse $\beta=1 / T$ should be understood as vectors (confirming Nakamura, 2009, who suggested it for different reasons). In presence of anisotropy they become tensors. Including particle spins requires a slightly different treatment.

Acknowledgements. We thank the anonymous referee for pointing out an inconsistency in the original submission in the nonrelativistic limit. We thank P. H. Yoon for important remarks on the history and references.

The topical editor, E. Roussos, thanks P. Yoon and one anonymous referee for help in evaluating this paper.

\section{References}

Alves, E. P., Grismayer, T., Fonseca, R. A., and Silva, L. O.: Transverse electron-scale instability in relativistic shear flows, Physica A, 444, 963-969, doi:10.1016/j.physa.2015.09.100, 2015.

Chacón-Acosta, G., Dagdug, L., and Morales-Técotl, H. A.: Manifestly covariant Jüttner distribution and equipartition theorem, Phys. Rev. E, 81, 021126, doi:10.1103/PhysRevE.81.021126, 2010.

Curado, E. M. F., Germani, F. T. L., and Soares, I. D.: Search for a Lorentz invariant velocity distribution of a relativistic gas, Phys. Plasmas, 22, 055601, doi:10.1063/1.4919391, 2016.

De Vore, C. R., Antiochos, S. K., Black, C. E., Harding, A. K., Kalapothrarakos, C., Kazanas, D., and Timokhin, A. N.: A model for the electrically charged current sheet of a pulsar, Astrophys. J., 801, 109, doi:10.1088/0004-637X/801/2/109, 2015.

Gladd, N. T.: The whistler instability at relativistic energies, Phys. Fluids, 26, 974-982, doi:10.1063/1.86429 1983.

Jüttner, F.: Das Maxwellsche Gesetz der Geschwindigkeitsverteilung in der Relativitätstheorie, Ann. Phys., 339, 856-882, doi:10.1002/andp.19113390503, 1911a.

López, R. A., Moya, P. S., Muñoz, V., Viñas, A. F., and Valdivia, J. A.: Kinetic transverse dispersion relation for relativistic magnetized electron-positron plasmas with Maxwell-Jüttner velocity distribution functions, Phys. Plasmas, 21, 092107, doi:10.1063/1.4894679, 2014.

Nakamura T. K.: Relativistic equilibrium distribution by relative entropy maximization, Europhys. Lett. EPL, 88, 40009, doi:10.1209/0295-5075/88/40009, 2009.

Swisdak, M.: The generation of random variates from a relativistic Maxwellian distribution, Phys. Plasmas, 20, 062110, doi:10.1063/1.4812459, 2013.

Yoon, P. H.: Electromagnetic Weibel instability in a fully relativistic bi-Maxwellian plasma, Phys. Fluids B-Plasma, 1, 1336-1338, doi:10.1063/1.858961, 1989.

Yoon, P. H.: Relativistic Weibel instability, Phys. Plasmas, 14, 024504, doi:10.1063/1.2646285, 2007.

Zenitani, S.: Loading relativistic Maxwell distributions in particle simulations, Phys. Plasmas, 22, 042116, doi:10.1063/1.4919383, 2015.

\footnotetext{
${ }^{1}$ We thank P. H. Yoon for bringing this early reference to our attention.
} 